\title{
HIDDEN STATISTICS IN SPECKLE PATTERNS
}

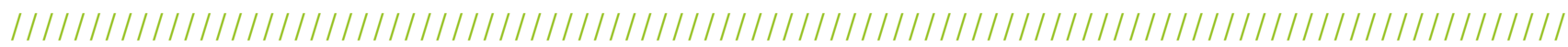

\section{Rémi CARMINATI}

Institut Langevin, ESPCI Paris, PSL University, CNRS, 75005 Paris, France

*remi.carminati@espci.psl.eu

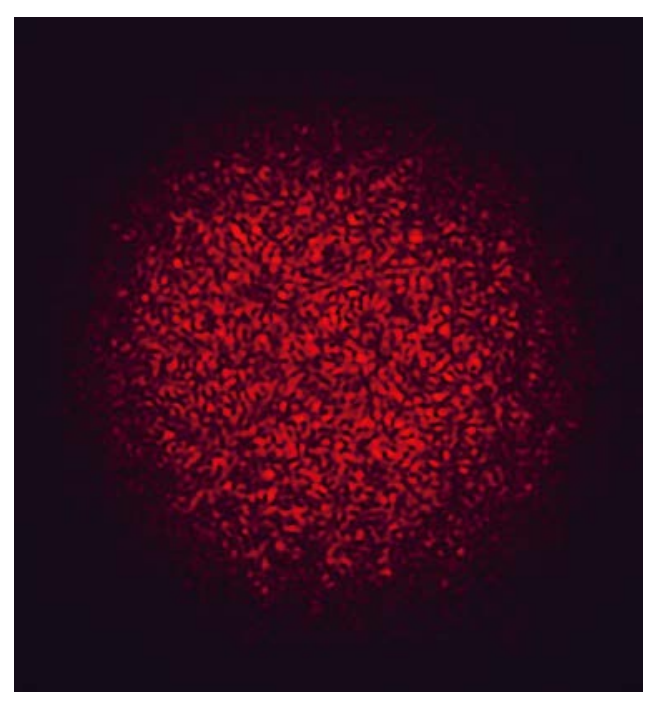

\begin{abstract}
Scattering of coherent light from a disordered material produces a complex distribution of intensity known as a speckle pattern. Speckle patterns are not as random as they appear at first glance. Their statistical properties exhibit universal features, as already recognized in the first paper on laser speckle. The existence of short-range and long-range spatial correlations in speckles has been shown to be fundamental in wave physics. It has also led to the emergence of novel approaches for imaging through scattering media.
\end{abstract}

https://doi.org/10.1051/photon/202111032

This is an Open Access article distributed under the terms of the Creative Commons Attribution License (http://creativecommons.org/licenses/by/4.0), which permits unrestricted use, distribution, and reproduction in any medium, provided the original work is properly cited.

\section{A HISTORICAL PAPER ON LASER SPECKLE}

In 1963, shortly after the laser was invented, Robert Langmuir reported on a curious phenomenon in a Letter entitled Scattering of Laser Light [1]. By illuminating a piece of paper with a helium-neon laser, he observed a pattern of dark and bright spots, which could be seen both in transmission and reflection. Without using the word, Langmuir described what we now call a speckle pattern, and his Letter seems to be the first report on laser speckle produced by scattering. After a brief qualitative description of the phenomenon, Langmuir assumes that the observed behaviour "must surely be related to the coherence and extreme monochromaticity of the light emitted from the laser". Using an analogy with radar wave propagation, he borrows a known result in this domain to deduce that the probability distribution of the intensity $I$ in the speckle pattern must be of the form $\exp \left(-I / I_{0}\right)$.

Although intuitively introduced by Langmuir in his paper, this result actually reveals the existence of fundamental statistical properties in speckle patterns. This negative exponential distribution, known as Rayleigh statistics, is more precisely written as $\exp (-I /\langle I\rangle) /\langle I\rangle$, where $\langle I\rangle$ is the average intensity. Its universal character, independent of the nature of the scattering object generating the speckle pattern, is remarkable. In fact it can be shown that the distribution follows from an application of the central-limit theorem, under very general assumptions that are quite robust in practice (see insert 1). 


\section{RAYLEIGH STATISTICS}

In a speckle pattern, formed by shining monochromatic light on a scattering medium, the complex field amplitude $E(r)$ is the superposition of partial waves emerging from all possible scattering paths inside the medium. Three of such paths are represented in the figure. In the simplest model, one assumes that the partial waves are statistically independent with their phases uniformly distributed (roughly speaking, the waves are "as random as possible") [2]. The amplitude $E(\boldsymbol{r})$ being the sum of a large number of independent random amplitudes, its real and imaginary parts follow a Gaussian statistical distribution by virtue of the central-limit theorem. The intensity $I(r)=|E(r)|^{2}$ therefore follows a negative exponential distribution, also known as Rayleigh statistics.

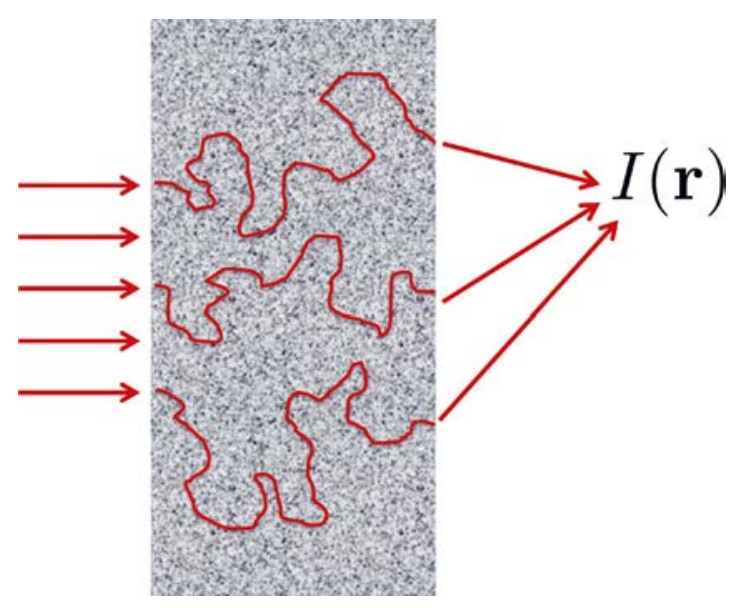

\section{NOISE OR SIGNAL?}

With the advent of the laser, the observation of speckle in optics has become commonplace, and integral to the development of coherent detection and imaging techniques. Many book chapters, and even dedicated textbooks, are devoted to speckle [2]. At first sight, the granularity of speckle creates some noise superimposed on signals or images, and the reduction of speckle noise becomes a key issue. However, speckle being interferometric in nature, its high sensitivity to the scattering medium can be used, for example, to detect a deformation or to probe the internal dynamics of a medium in which the scatterers are in motion (such as particles in suspension in milk or a blood flow). In this case a statistical property is used, such as a spatial or temporal correlation function of the detected intensity, to measure a parameter on which it depends (in this case, the chosen statistical property has to be non-universal). From noise, the speckle becomes a signal.

\section{SPECTROGON}

Interference filters

200 to $15000 \mathrm{~nm}$

- Bandpass

- Longwave-pass

- Shortwave-pass

- Broad-bandpass

- Neutral density

- Web stock items

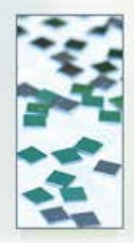

UK: sales.uk@spectrogon.com·Tel+44 1592770000 Sweden (headquarters): sales se@spectrogon com • Tel +46 86382800 US: sales.us@spectrogon.com·Tel +19733311191
Holographic gratings

150 to $2000 \mathrm{~nm}$

- Pulse compression

- Telecom

- Laser tuning

- Monochromator

- Spectroscopy

- Web stock items 


\section{ORIGIN OF LONG-RANGE CORRELATIONS IN SPECKLE PATTERNS}

(a)

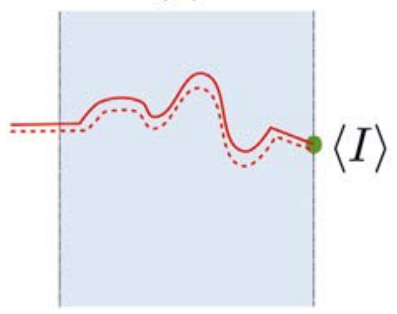

(b)

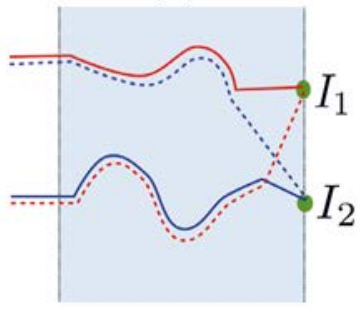

(c)

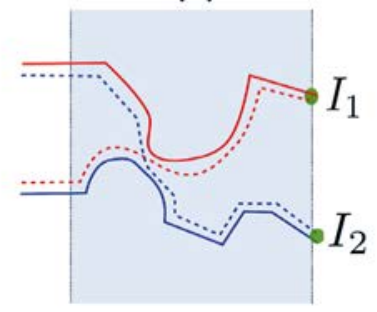

(d)

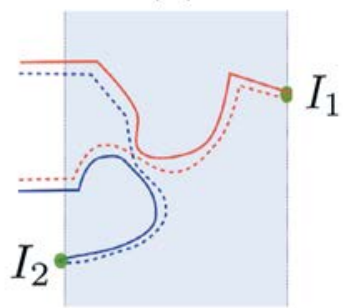

A wave in a disordered medium propagates along scattering paths, schematically represented as wavy lines. To form the intensity $I=|E|^{2}$, one needs to pair two scattering paths corresponding to $E$ (shown as a solid line) and to its complex conjugate (shown as a dashed line). In multiple scattering theory, one shows that the leading term in the average intensity $<1>$ corresponds to a field and its complex conjugate following the same scattering paths (other pairings vanish after averaging due to destructive interferences) [6]. One uses the representation in (a) for the average intensity, with the double line known as a "ladder". Observing now the intensities $l_{1}$ and $l_{2}$ at two different points, a correlation between them can be created by crossings between ladders (two independent ladders would simply lead to $\left\langle l_{1} I_{2}\right\rangle=\left\langle l_{1}\right\rangle\left\langle l_{2}\right\rangle$ ). If the crossing occurs at the last scattering event, followed by ballistic propagation towards the observation points as represented in (b), one creates a short-range correlation characterizing the size of the speckle spots. If the crossing occurs deep inside the medium, followed by diffusion as shown in (c), one creates long-range correlations. This mechanism can also generate intensity correlations between two observation points on opposite sides of the scattering medium, in the reflected and transmitted speckle patterns. This kind of correlation has been observed only recently [7].

\section{MULTIPLE SCATTERING \\ AND THE CONNECTION \\ TO MESOSCOPIC PHYSICS}

The traditional description of speckle in optics has long been based on a physical optics approach, assuming a single scattering regime, in which the different points of the object are illuminated by the incident wave and scatter towards the detector, without interaction between them. From the mid-1980s, the emergence of multiple light scattering as a full-fledged research topic gave a second wind to the study of optical speckles. This emergence was stimulated on the one hand by applications in imaging through strongly scattering media (such as biological tissues), and on the other hand by fundamental questions in mesoscopic physics. Indeed, the expected analogies between quantum transport of electrons in condensed matter, and multiple scattering of coherent light in disordered media, have allowed to revisit the statistical properties of optical speckles.

Figure 1. Measurement of the reflected and transmitted speckle patterns produced on both sides of a scattering medium. A statistical correlation between them persists even when the medium is opaque due to multiple scattering. Adapted from [7].
The spatial correlation function of the intensity in a three-dimensional speckle has been predicted, showing a universal short-range component characterizing the average size of a speckle grain [3]. More surprisingly, the existence of long-range intensity correlations (well beyond the size of a speckle grain) has also been predicted [4]
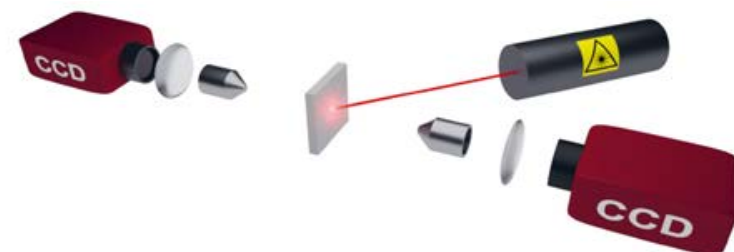

Reflected speckle

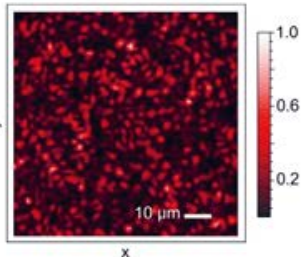

Transmitted speckle

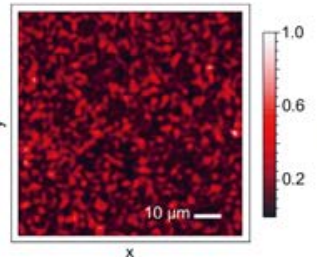

Correlation function

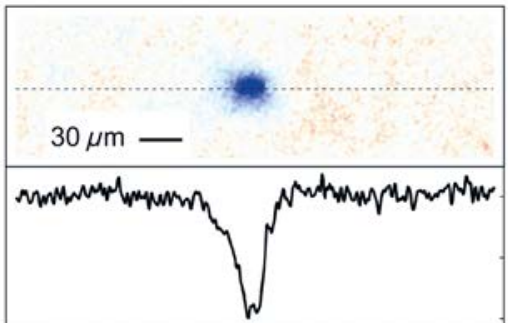




\section{When your phase has to}

(see insert 2). These long-range correlations can be demonstrated by measuring the intensity correlation function at two different points, or at two different times or frequencies [5], which is possible in optics but not for electrons in condensed matter. The study of optical speckles became more and more fundamental in wave physics, with an impact far beyond the field of optics.

\section{MORE SURPRISES WITH LONG-RANGE SPECKLE CORRELATIONS}

In principle, the mechanism responsible for the long-range correlations in a speckle pattern should allow one to generate correlations between speckle patterns measured in reflection and transmission on both sides of a turbid scattering medium (see panel (d) in insert 2). After a theoretical study showed the possibility, the existence of such a statistical link has been demonstrated experimentally [7]. The experiment and the result are summarized schematically in figure 1 . The existence of a statistical correlation between the intensities measured at two points on either side of the scattering medium proves that the reflected and transmitted speckle patterns share mutual information, even in a multiple scattering regime in which the medium is opaque (meaning that it is not possible to form a direct image through it).

Sometimes noise, sometimes signal, object of fundamental studies or tool for new optical techniques, speckle patterns have a long history. Today, the possibility to manipulate them using wavefront control techniques, and the use of their longrange statistical correlations, have opened new avenues for imaging and detection in or through strongly scattering media. A long story, therefore, and far from over.

\section{REFERENCES}

\section{[1] R.V. Langmuir, Appl. Phys. Lett. 2, 29 (1963)}

[2] Laser Speckle and Related Phenomena, edited by C. Dainty (Springer-Verlag, Berlin, 1975); J.W. Goodman, Speckle Phenomena in Optics (Roberts and Company, Greenwood Village, 2007)

[3] B. Shapiro, Phys. Rev. Lett. 57, 2168 (1986)

[4] S. Feng, C. Kane, P.A. Lee and A.D. Stone, Phys. Rev. Lett. 61 , 834 (1988)

[5] J. F. de Boer, M. P. van Albada, and A. Lagendijk, Phys. Rev. B 45, 658 (1992); F. Scheffold and G. Maret, Phys. Rev. Lett. 81, 5800 (1998)

[6] R. Carminati and J.C. Schotland, Principles of Scattering and Transport of Light (Cambridge University Press, Cambridge, 2021)

[7] I. Starshynov, A. M. Paniagua-Diaz, N. Fayard, A. Goetschy, R. Pierrat, R. Carminati, and J. Bertolotti, Phys. Rev. X 8, $021041(2018)$

\section{travel on time}

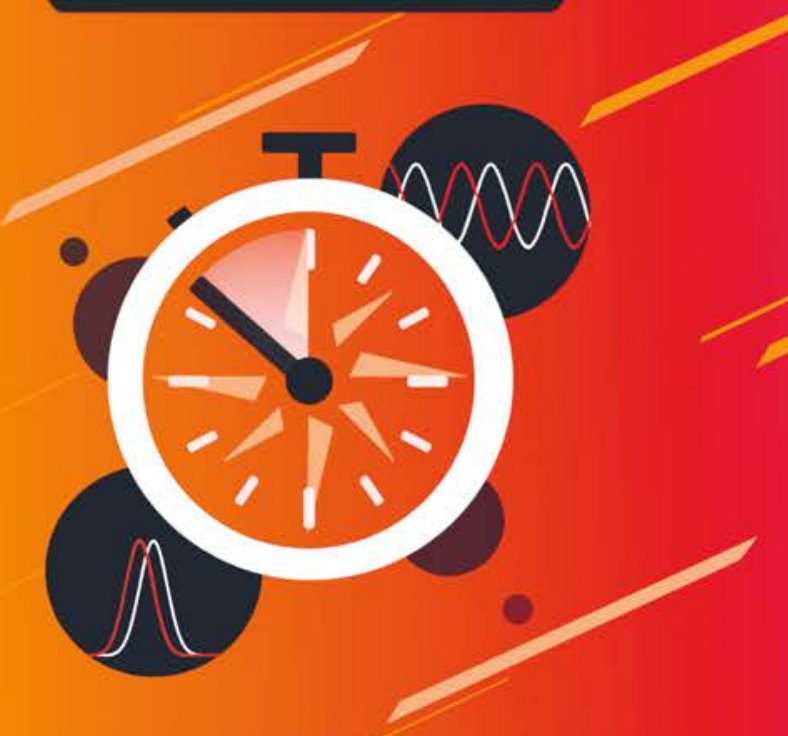

FULL RANGE OF DELAY MANAGEMENT SOLUTIONS

[ Available during Q4 2021]

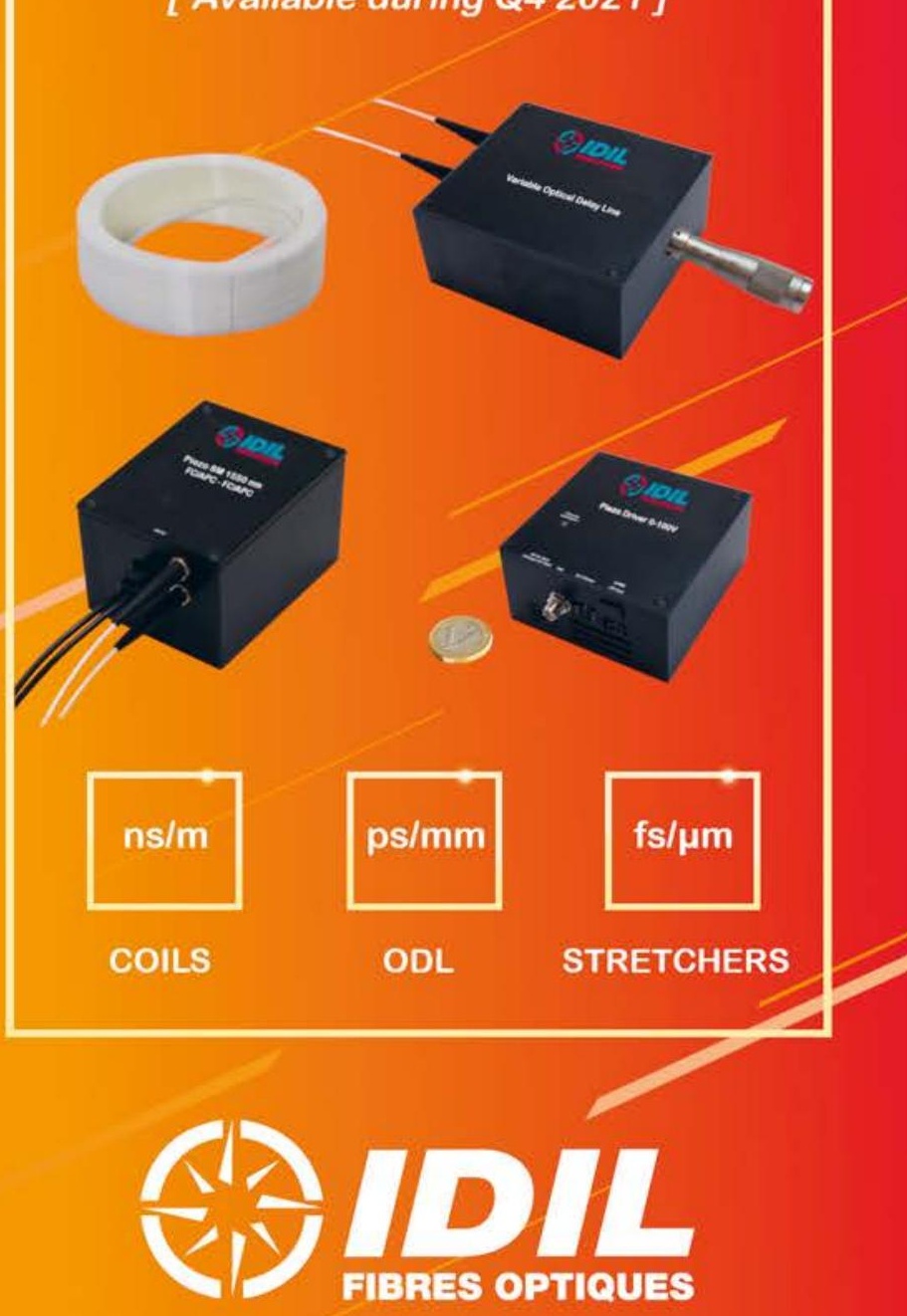

w w w i d i I. f r 